\title{
Mechanical crosstalk between vertical and lateral piezoresponse force microscopy
}

\author{
F. Peter, ${ }^{\text {a) }}$ A. Rüdiger, ${ }^{\text {b) }}$ and R. Waser \\ Institut für Festkörperforschung (IFF), Forschungszentrum Jülich, 52425 Jülich, Germany and Center of \\ Nanoelectronic Systems for Information Technology (cni), Forschungszentrum Jülich, 52425 Jülich, Germany
}

(Received 24 November 2005; accepted 23 January 2006; published online 16 March 2006)

\begin{abstract}
Piezoresponse force microscopy (PFM) provides valuable insight into the inverse lateral and vertical piezoelectric effects on the nanoscale. Ideally, these contributions are separated into vertical and lateral detections of a deflected laser beam on a quadrupole diode. In contrast to the known crosstalk by a rotated diode that causes identical signals in both channels, we report on the crosstalk due to the geometrical constraints of the cantilever that is inherent to the lateral PFM. For a $\mathrm{BaTiO}_{3}(001)$ nanograin we show that the vertical response attributable to the crosstalk is $1 / 8$ th of the lateral response. From this value we deduce the actual mechanism responsible for the crosstalk. (C) 2006 American Institute of Physics. [DOI: 10.1063/1.2176081]
\end{abstract}

Piezoresponce force microscopy (PFM) has become an indispensable tool in the investigation of piezoelectric materials. ${ }^{1-4}$ As soon as laterally confined structures, instead of continuous thin films, are under investigation, a careful distinction between extrinsic influences and the actual piezoresponse becomes mandatory. In a previous Letter we have shown the influence of grain topography on lateral piezoresponse measurements, ${ }^{5}$ essentially giving rise to an enhancement of the lateral piezoelectric response along the perimeter. However, this enhancement is more pronounced in the direction perpendicular to the cantilever axis ${ }^{6}$ (usually identical with the scanning direction). As a lateral movement of the cantilever tip parallel to the cantilever axis causes a bending instead of a torsion, the respective signal is monitored in the channel for vertical movements. As the optical amplification of the vertical channel can be up to a factor of 18 below that of the lateral channel for a commercially available cantilever (with length of $450 \mu \mathrm{m}$ and tip height of $15 \mu \mathrm{m}),{ }^{6,7}$ this crosstalk is hard to detect. In most modern atomic force microscopes (AFM) the position of the cantilever is detected by means of a four quadrant photodiode. A laser beam is reflected from the top of the cantilever. If the cantilever bends up or down, the reflected beam will be deflected up or down. The torsion of the cantilever deflects the laser to the left or the right. One problem of this setup occurs if the photodiode is rotated and thereby not in the same plane as the cantilever. As a result, a lateral motion of the laser spot will cause the vertical signal to change. ${ }^{8}$ As long as the photodiode cannot be rotated or other system modifications are undertaken to eliminate this effect, ${ }^{9,10}$ this crosstalk is present in all types of measurements. While this crosstalk is known to affect any kind of atomic force microscope measurements with lateral sensitivity, ${ }^{11}$ another kind of crosstalk with a completely different physical origin exists in PFM.

Figure 1(a) depicts part of the general PFM setup where

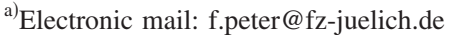

b)Electronic mail: a.ruediger@fz-juelich.de
}

a piezoelectric grain is electrically contacted by a conducting cantilever. If an ac voltage is applied to the cantilever and the substrate is grounded, the grain expands and contracts due to the inverse piezoelectric effect. The amplitudes of the $x, y$, and $z$ displacements only depend on the piezoelectric coefficients of the material. However, these deformations in the different directions cannot be detected independently. Especially, due to the symmetry of the cantilever the lateral piezoresponse can only be detected on the grain slopes parallel to the cantilever axis. In Fig. 1(b) these are positions $b$ and $d$. Part a of Fig. 2 shows a deformation of the grain in the $y$ direction. The cantilever is twisted, resulting in a movement of the laser on the photodiode in the lateral direction. This is the normal case for the lateral PFM. The mechanical crosstalk can be seen in Fig. 2(b). In the sketch we only consider the deformation in the $x$ direction. Although this is clearly a lateral deformation, due to the geometry of the cantilever, it leads to a vertical movement of the cantilever. This movement is detected as a vertical displacement of the laser on the photodiode. A vertical response is recorded, even though its physical origin is lateral.

The question arises if this extrinsic piezoelectric effect is sufficiently pronounced to be experimentally detected. We analyze this on a (001) orientated $\mathrm{BaTiO}_{3}$ grain prepared by pulsed laser deposition on $\mathrm{SrRuO}_{3}(50 \mathrm{~nm})$ covered (100) $\mathrm{SrTiO}_{3}$ single crystal. The PFM measurements are done on an enhanced Jeol 4210 AFM. A voltage of $U=5 \mathrm{~V}$ at $7 \mathrm{kHz}$ is applied to a PtIr $_{5}$ coated cantilever ("ContPt" from Nanosensors, $f_{0}=13 \mathrm{kHz}$ ).

Figure 3 shows the topography as well as the lateral and vertical piezoresponses of this sample. The situation described in the previous section can be clearly identified: in the lateral PFM scan (part (c)] the maximum amplitude is at positions $b$ and $d$ with practically no response at positions $a$ and $c$. Figure 3(b) depicts the vertical movement of the cantilever; a significant response can only be seen in positions $a$ and $c$ which is the crosstalk from the lateral response.

Two different physical effects can be the cause of this 
(a)
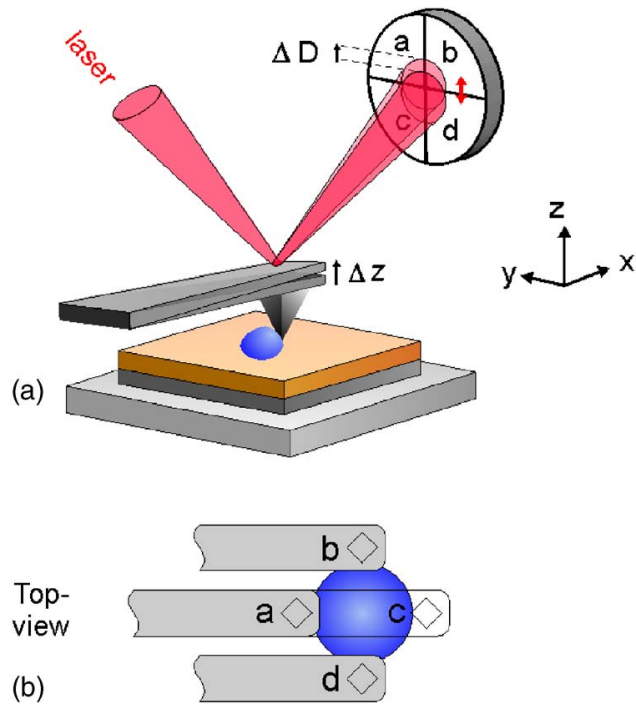

FIG. 1. (Color online) (a) Piezoelectric nanograin, conducting cantilever, laser, and photodiode as arranged in a PFM setup; (b) top view of the cantilever and the grain indicating the different positions.

crosstalk. In the first case depicted in Fig. 4(a), the cantilever tip "sticks" to the grain. When the grain expands along $-x$, the cantilever has to be stretched, resulting in a tilting of the tip, i.e., a detectable vertical signal. Taking the dimensions of the setup (cantilever length of $450 \mu \mathrm{m}$ and expansion of the grain a few picometer) into account, we assume that the center of the tip [point $P$ in Fig. 4(a)] remains stationary. As this situation is geometrically equivalent to the lateral response, ${ }^{12}$ the ratio

$$
R=\frac{\text { lateral amplitude }}{\text { vertical amplitude }}
$$

(a)
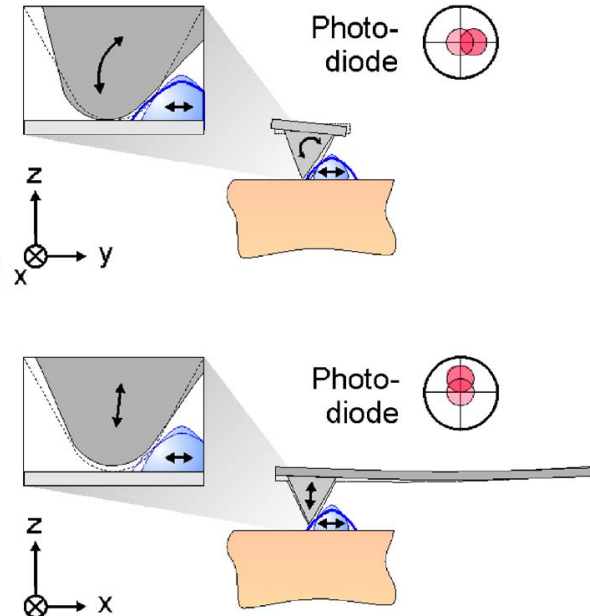

(b) $y$

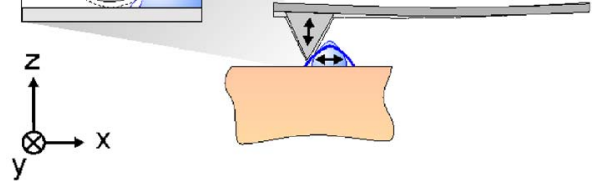

FIG. 2. (Color online) (a) Analysis of the cantilever movement for an expansion of the grain in direction $y$ and (b) analysis of the cantilever movement for an expansion of the grain in direction $x$ resulting in a mechanical crosstalk.
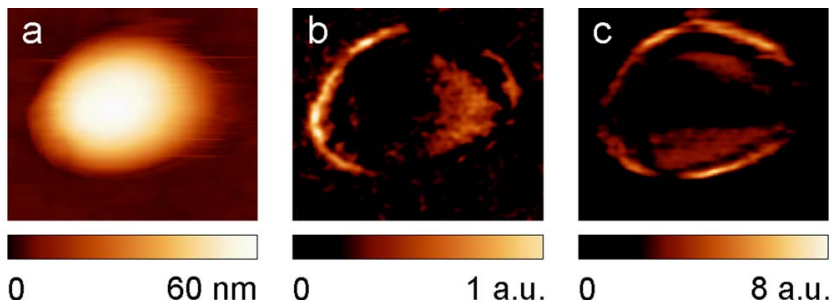

1 a.u.

8 a.u.

FIG. 3. (Color online) (a) Topography $\left(600 \times 500 \mathrm{~nm}^{2}\right)$, (b) vertical piezoresponse amplitude, and (c) lateral piezoresponse amplitude. The amplitude of the lateral response is eight times larger than the vertical response. Note that in the vertical case the amplitude maximum is on the left and the right of the grain, whereas in the lateral case it is on the top and the bottom.

should be almost unity and should not differ by nearly one order of magnitude, as observed. In the second scenario [Fig. 4(b)] the tip slips over the grain. An expansion of the grain along $-x$ thus leads to a lifting of the tip and the cantilever. Here we would expect the ratio of the amplitudes to be $R=18$. ${ }^{6}$ The slope of the grain modifies this consideration as it translates the lateral expansion into a vertical deflection of the cantilever. In Fig. 3(a) we observe a maximum inclination angle of $65^{\circ}$ at position $a$ of the grain. According to

$$
\frac{\text { vertical deflection }}{\text { lateral expansion }}=\frac{\nu}{l}=\tan \varphi
$$

that provides an additional factor of 0.47 to the ratio $R$ i.e., $R^{\prime}=18 \tan 65^{\circ} \approx 8.4$. This is within a $5 \%$ experimental error of the observed value. From this quantitative agreement we deduce that the scenario as depicted in Fig. 4(b) is the appropriate description.
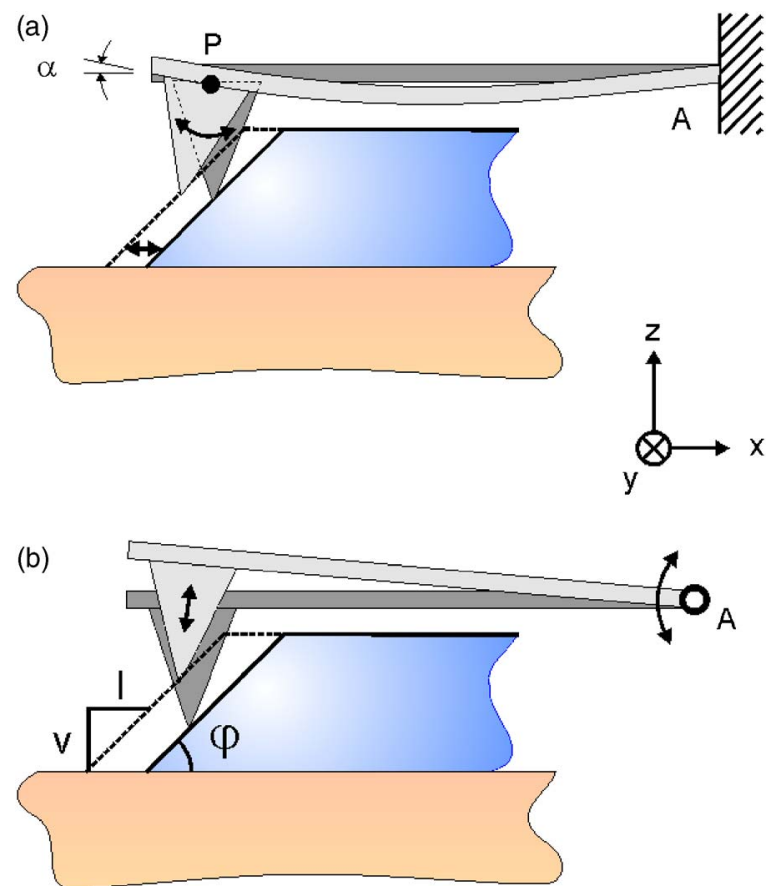

FIG. 4. (Color online) Two different situations resulting in a mechanical crosstalk. (a) The cantilever tip "sticks" to the grain and has to be stretched when the grain expands. This results in a tilting of the tip (angle $\alpha$ ). (b) The tip slips over the grain, and an expansion of the grain leads to a lifting of the tip. The measured values validate that the second case is the dominating one. The images are not drawn to scale. 
An explanation why a lateral signal can at all be detected despite a (001) orientation is given in Refs. 5 and 6. The crosstalk has a tremendous relevance for the investigation of size effects in ferroelectric nanostructures, as the observed additional vertical deflection of the laser beam is not caused by a modified polarization. According to Ref. $13, d_{33}$ (causing the vertical expansion) is directly proportional to the polarization (in samples with a cubic paraelectric phase which is true for $\mathrm{BaTiO}_{3}$ ). In that sense our experiment confirms that no size effect has so far been observed due to lateral scaling in ferroelectric nanostructures.

In this Note we have shown the existence of mechanical crosstalk between lateral and vertical PFM. This crosstalk is inherent to the scanning method and occurs in addition to the misalignment crosstalk of the AFM photodetector. Measurements show that this effect can be substantial when analyzing piezoelectric grains. We can conclude that the observed enhancement of the lateral signal is not due to a corresponding enhancement of the piezoelectric tensor element $d_{33}$ but merely an inevitable geometrical consequence of the setup.
The authors would like to thank Regina Dittmann for providing the $\mathrm{BaTiO}_{3}$ sample.

${ }^{1}$ L. M. Eng, H.-J. Güntherodt, G. Rosenman, A. Skliar, M. Oron, M. Katz, and D. Eger, J. Appl. Phys. 83, 5973 (1998).

${ }^{2}$ A. Roelofs, U. Böttger, R. Waser, F. Schlaphof, S. Trogisch, and L. M. Eng, Appl. Phys. Lett. 77, 3444 (2000).

${ }^{3}$ A. Gruverman, O. Auciello, and H. Tokumoto, J. Vac. Sci. Technol. B 14, 602 (1996).

${ }^{4}$ M. Alexe, C. Harnagea, D. Hesse, and U. Gösele, Appl. Phys. Lett. 79, 242 (2001).

${ }^{5}$ F. Peter, A. Rüdiger, R. Dittmann, R. Waser, K. Szot, B. Reichenberg, and K. Prume, Appl. Phys. Lett. 87, 082901 (2005).

${ }^{6}$ F. Peter, A. Rüdiger, K. Szot, and R. Waser (in press).

${ }^{7}$ This valve is corrected by a factor of 2 as compared to Ref. 13 .

${ }^{8}$ R. Piner and R. S. Ruoff, Rev. Sci. Instrum. 73, 3392 (2002).

${ }^{9}$ M. Varenberg, I. Etsion, and G. Halperin, Rev. Sci. Instrum. 74, 3569 (2003).

${ }^{10}$ M. Such, D. E. Kramer, and M. Hersam, Ultramicroscopy 99, 189 (2004).

${ }^{11}$ S. Jeon, Y. Braiman, and T. Thundat, Rev. Sci. Instrum. 75, 4841 (2004).

${ }^{12}$ F. Peter, A. Rüdiger, R. Waser, K. Szot, and B. Reichenberg, Rev. Sci. Instrum. 76, 046101 (2005).

${ }^{13}$ A. L. Kholkin, K. G. Brooks, and N. Setter, Appl. Phys. Lett. 71, 2044 (1997). 